\title{
Experimental leprosy in rhesus monkeys: transmission, susceptibility, clinical and immunological findings
}

\author{
BOBBY J. GORMUS, KEYU XU, GARY B. BASKIN, \\ LOUIS N. MARTIN, RUDOLF P. BOHM, JR, \\ JAMES L. BLANCHARD, PAMELA A. MACK, \\ MARION S. RATTERREE, WAYNE M. MEYERS* \\ \& GERALD P. WALSH† \\ Departments of Microbiology, Pathology and Veterinary Sciences, \\ Tulane Regional Primate Research Center, 18703 Three Rivers \\ Road, Covington, LA 70433, USA; *Armed Forces Institute of \\ Pathology, Washington, DC 20306-6000, USA; and †American \\ Leprosy Foundation, 11600 Nebel Street, Suite 210, Rockville, \\ MD 20852, USA
}

\section{Accepted for publication 29 May 1998}

\begin{abstract}
Summary A total of 46 Rhesus monkeys (RM) was inoculated with Mycobacterium leprae (ML) and followed clinically and immunologically for extended periods. Twenty-one $(45.7 \%)$ of the RM developed leprosy spanning the known leprosy spectrum, with six of $21(28.6 \%)$ having disease in the borderline lepromatous to lepromatous area of the spectrum. RM with paucibacillary forms of leprosy produced predominantly IgG anti-phenolic glycolipid (PGL-I) antibodies and positive lepromin skin test and/or in vitro blastogenesis responses; IgM anti-PGL-I predominated in animals with BB-LL leprosy and correlated with negative immune responses to lepromin. IgG anti-PGL-I antibodies persisted in a number of RM for several years without histopathological evidence of leprosy, suggesting possible persisting subclinical infection. The data show that RM are a valuable model for the study of leprosy. Eleven of the $46 \mathrm{RM}$ were inoculated with $\mathrm{ML}$ from sources infected with simian immunodeficiency virus (SIV), the monkey counterpart to the human immunodeficiency virus (HIV). The possible effect of SIV on the clinical outcome of ML infection could not be determined due to insufficient numbers of animals to yield statistically significant results.
\end{abstract}

\section{Introduction}

We previously reported that it is possible experimentally to transmit leprosy to rhesus monkeys (RM) (Macaca mulatta) and cynomolgus monkeys (CM) (Macaca fascicularis). ${ }^{1-3}$

Correspondence to: B. J. Gormus 
Prior to those reports, attempts experimentally to transmit leprosy to RM and other macaque species were notably unsuccessful, although many experimental inoculations had been made over approximately a century. ${ }^{4}$ In addition to our prior success in experimental transmission of leprosy to a small number of RM and CM, we also observed three feral CM with positive leprosy serology, ${ }^{3}$ and more recently, a case of natural leprosy was diagnosed in a wildcaught CM from the Philippines (Canfield et al., personal communication). To our knowledge, this case of natural leprosy and the previous three CMs with positive leprosy serology, which remain under observation, are the only known reported examples of natural leprosy in macaque species. Herein, we report data from experimental inoculations of a total of 46 RM. ML inocula were taken from sooty mangabey monkeys (SMM) with lepromatous (LL) leprosy or from armadillos that had been experimentally inoculated with ML from SMM or human sources. The results extend our earlier observations that RM are susceptible to experimentally induced leprosy, and permit a comparison of the susceptibility of RM to humans and that of other primate species. ${ }^{1-6}$

\section{Materials and methods}

\section{INOCULATIONS}

Lepromata (see Table 1 for sources) were obtained aseptically, minced and homogenized in cold phosphate-buffered saline using a Dounce homogenizer with a 40 MI mortar and teflon pestle (Wheaton Scientific, Millville, NJ, USA), passed through gauze and centrifuged at $200 \mathrm{~g}$ for $5 \mathrm{~min}$ at $4^{\circ} \mathrm{C}$. The acid-fast bacilli (AFB) in the supernatant were counted and morphological indices (MI) determined by the method of Shepard \& McCrae. ${ }^{7} \mathrm{RM}$ were inoculated with ML suspensions by combined intradermal (ID) and intravenous (IV) routes using two ID sites per ear, the tip of the nose, lateral forearms and lateral calves. IV inoculations were made via the saphenous vein.

\section{CLINICAL OBSERVATIONS}

Animals were observed twice daily and examined in detail three or four times per year or more, depending on the status of the animal. Clinical aspects of the disease were recorded at each time of observation. The Ridley-Jopling system was used to classify leprosy histopathologically, ${ }^{8}$ with the exception that classification at the paucibacillary end of the spectrum differs slightly in RM from humans. The Ind/TT (indeterminate/tuberculoid) classification has been included to describe a well-defined grossly tuberculoid-appearing

Table 1. ML inoculation of $\mathrm{RM}^{\mathrm{a}}$

\begin{tabular}{lccc}
\hline No. of RM inoculated & ML origin & ML dose $\left(\times 10^{-8}\right)$ & No. of RM with leprosy \\
\hline 23 & SMM/A & $1-10$ & 12 \\
11 & SMM & $1-10$ & 6 \\
12 & HU/A & $50-1000$ & 3 \\
\hline
\end{tabular}

\footnotetext{
${ }^{\text {a }}$ Abbreviations: ML, M. leprae; RM, rhesus monkeys; SMM, sooty mangabey monkeys; $\mathrm{Hu}$, human; and A, armadillo.
} 
lesion with rare $\mathrm{AFB}$, usually in nerves, and minimal to mild perivascular mononuclear inflammatory cell infiltrate. Often the skin lesion is dry, scaly and hypopigmented with alopecia. No histiocytes, epithelioid cells or multinucleated cells are present. TT leprosy by classical histopathological definitions, containing epithelioid and/or multinucleated giant cells together with lymphocytes, is less frequently observed, but does occur in RM. The terms neuritic TT and neuritic Ind/TT are used herein to describe lesions with predominantly neuritic involvement.

\section{ELISA}

The assays were performed as previously reported. ${ }^{9-12}$ Baseline sera were obtained prior to ML inoculations and at intervals after inoculation, and were stored frozen for later ELISA evaluations of ML-specific anti-PGL-I antibody. Natural ML PGL-I was used as antigen $(\mathrm{Ag})$ [specificity for PGL-I was verified using the synthetic glycoconjugate, bovine serum albumin- $O$-(3,6-di- $O$-methyl- $\beta$-D-glucopyranosyl)-(1-4)-(1-deoxy-2,3-di- $O$-methyl-Lrhamnose) (NDO-BSA), instead of natural PGL-I in all animals; only the natural PGL-I data are reported]. PGL-I and ND0-BSA were provided by Dr Patrick J. Brennan, Colorado State University School of Veterinary Medicine, Fort Collins, CO, USA under NIH contract no. 1Al-52582.

Briefly, 96-well plates were coated with Ag, washed, blocked with BSA, washed again and reacted with a previously determined optimal dilution of monkey serum (1:150 for IgG and 1:100 for anti-IgM). After incubation and washing, the plates were coated with peroxidase-labelled anti-human IgG or IgM Fc fragment $\gamma$ - or $\mu$-chain-specific Ab diluted according to prior titrations, incubated, washed, reacted with $o$-phenylenediamine $+\mathrm{H}_{2} \mathrm{O}_{2}$, acidified, and optical density (OD) determined at $490 \mathrm{~nm}$ on an ELISA reader. Final ODs represent the difference in absorbance between wells containing Ag minus wells lacking Ag but containing all other components. Each reagent in the ELISA was carefully titrated in a checkerboard manner to determine dilutions that would give final OD values between $0 \cdot 1$ and $0 \cdot 5$ OD whenever possible, enabling the utilization of an OD range most sensitive to small changes in OD, so that small changes from sample to sample would have maximal meaning and would accurately reflect longitudinal changes. All sera were assayed together at one time in given experiments to permit accurate relative comparisons. The same batch of peroxidase antibody was used throughout. All experiments were repeated on at least two separate occasions, examining all sera together in each assay. OD values obtained with these precautions were reproducible in a given sample from one assay to another to within $\pm 0 \cdot 05$. Cut-off points for positivity were taken as OD values greater than the mean +2 standard deviations (SD) of the mean of normal RM $(n=73)$. The mean +2 SD cut-off points were $0.018+0 \cdot 114(0 \cdot 132)$ for IgG and $0.017+0.094(0 \cdot 111)$ for IgM anti-PGL-I.

Ratios of ELISA values for IgM:IgG were calculated in some instances, as noted in the text, by simply dividing the actual IgG OD value into the IgM OD value, as previously described. ${ }^{9-12}$

\section{LEPROMIN SKIN TESTING}

RM require 10 times the standard human concentration of lepromin (lepromin A) to induce responses consistently. ${ }^{6}$ A concentrated $(\times 76 \cdot 9)$ lepromin A suspension in PBS was prepared by Dr Wayne M. Meyers at the Armed Forces Institute of Pathology for use in these 
experiments. The stock was stored frozen and diluted with PBS just prior to use for skin testing. Animals were tested on the shaved lower abdomen by intradermally injecting $0 \cdot 1 \mathrm{Ml}$ of $(\times 10)$ lepromin $\left(1.6 \times 10^{9} \mathrm{M}\right.$. leprae $\left./ \mathrm{Ml}\right)$. The reaction size and characteristics were noted and biopsied on day 28 postinjection (Mitsuda reaction). Animals were skin tested between 1 and 2 years after ML inoculation. Results are according to WHO criteria: -, no reaction; +/- <4 mm; +, 4-6 $\mathrm{mm} ;++, 7-10 \mathrm{~mm}$ and,$+++>10 \mathrm{~mm}$.

\section{BLASTOGENESIS}

Heparinized blood was used to prepare buffy coats which were centrifuged on Ficoll/ Hypaque, washed and suspended in RPMI-1640 + 5\% heat-inactivated human AB serum (HuABS) and penicillin/streptomycin. The mononuclear cell (MNC) fraction was used for in vitro blastogenesis studies, using an antigen the same lepromin as that used for skin testing. A stock suspension of $\times 76.9$ lepromin was prepared for blastogenesis by 1:50 dilution into RPMI-1640 + 5\% HuABS. A final concentration of 1:20,000 of the 1:50 stock per well reproducibly gave optimal stimulations in preliminary testing and was used throughout this study. U-bottom 96-well microtitre plates were used. Aliquots of $2 \times 10^{5} \mathrm{MNC}$ per well were incubated at $37^{\circ} \mathrm{C}$ in $5 \% \mathrm{CO}_{2}$ in triplicate for 5 days with lepromin or media prior to pulsing for $4 \mathrm{~h}$ with $1 \mu \mathrm{CI}$ of ${ }^{3} \mathrm{H}$-thymidine/well. Thereafter, cells were washed and harvested on a cell harvester and quantified by scintillation counting. Stimulation indices (SI) were determined by subtracting backgrounds from all values and dividing the media control (C) values into the lepromin-stimulated experimental $(\mathrm{E})$ values $(\mathrm{SI}=\mathrm{E} / \mathrm{C})$. Positive SIs were greater than the mean $+2 \mathrm{SD}$ of the response of normal RM. In vitro lepromin blastogenesis studies were completed immediately prior to lepromin skin testing. The mean $+2 \mathrm{SD}$ cut-off value for lepromin blastogenic responses was $1 \cdot 66+3 \cdot 12(4 \cdot 78)$.

\section{Results}

CLINICAL

A total of $46 \mathrm{RM}$ were inoculated with ML from a variety of sources over a period of 11 years (Table 1); 21 (45.7\%) developed histopathologically documentable leprosy, including nine indeterminate (Ind)/tuberculoid (TT) (Ind/TT), four neuritic TT, two TT, two borderline (BB), one borderline lepromatous (BL) and three lepromatous (LL) forms. Thirty-four of the $46 \mathrm{RM}$ received by combined IV/ID routes a titrated range of $1 \times 10^{8}$ to $1 \times 10^{9} \mathrm{SMM}$-origin ML [either after subpassage through armadillos (23 RM) or direct from a lepromatous SMM (11 RM)]. An additional $12 \mathrm{RM}$ received titrated amounts ranging from $5 \times 10^{9}$ to $1 \times 10^{11}$ armadillo subpassaged human ML by similar combined IV/ID routes (Table 1). The MIs of the various inocula ranged from 7 to $21 \%$.

These inoculations were performed before it was learned that most captive SMM carry simian immunodeficiency virus (SIV) asymptomatically and that SIV is transmissible to other primates experimentally or by contact with SIV-contaminated body fluids such as ML inoculum prepared from the tissues of SMM. ${ }^{14,15}$ The ML inoculum was taken from SMM found to be SIV-positive. ${ }^{15}$ Although no systematic approach was taken to investigate the role of SIV in leprosy in these studies, observations regarding the SIV status of the ML inocula are summarized for clarification (Table 2). SIV does not cause demonstrable AIDS-like disease in armadillos; virus-culture and PCR studies show that tissues from armadillos inoculated 
Table 2. Clinical results of RM inoculated with SIV + or SIV $-\mathrm{ML}^{\mathrm{a}}$

\begin{tabular}{|c|c|c|c|c|c|}
\hline & \multirow{2}{*}{$\begin{array}{c}\text { RM inoculated } \\
\text { no. }\end{array}$} & \multicolumn{2}{|c|}{ Leprosy + } & \multicolumn{2}{|c|}{ BB-LL leprosy } \\
\hline & & no. & $(\%)$ & no. & $(\%)^{\mathrm{b}}$ \\
\hline SIV+ & 11 & 6 & $(54 \cdot 5)$ & 3 & $(50)$ \\
\hline SIV- & 35 & 15 & $(42 \cdot 8)$ & 3 & (20) \\
\hline Totals & 46 & 21 & $(45 \cdot 7)$ & 6 & $(28.6)$ \\
\hline
\end{tabular}

\footnotetext{
${ }^{a}$ ML contaminated with SIV (SIV+) or uncontaminated (SIV-).

${ }^{\mathrm{b}}$ Percentage of leprosy cases.
}

with SIV + ML are negative for SIV and SIV-derived RNA (Murphey-Corb. M. and Gormus, B. J., unpublished observations).

\section{IMMUNOLOGICAL CORRELATIONS}

As we have previously documented in other primate species, the presence of IgM anti-PGL-1 serum antibody correlated longitudinally with susceptibility to LL forms of leprosy (no. 1, F185; no. 2, A125; no. 3, F179 and no. 7, F187, Table 3) with only rare exceptions (no. 3, B465, Table 3). RM F179 (no. 3) initially developed Ind/TT-type leprosy and, at that time, had only IgG anti-PGL-I antibody in significant quantities (IgM:IgG ELISA ratio <1), but later the disease evolved into LL-type leprosy along with an evolution towards predominantly IgM anti-PGL-I (IgM:IgG ELISA ratio >1 (Table 3). This pattern of a high IgM:IgG antiPGL-I serum antibody ratio in multibacillary leprosy-susceptible animals was previously observed in SMMs ${ }^{9,10,12}$ chimpanzees ${ }^{16}$ and RM A125. ${ }^{6}$ RM F187 (no. 7, Table 3) initially developed LL leprosy with predominantly IgM anti-PGL-I serum antibody (IgM:IgG ratio $>1$ ), but eventually upgraded to Ind/TT leprosy involving predominantly dermal nerves (neuritic leprosy). The evolution of disease in F187 from LL to Ind/TT leprosy was paralleled by a longitudinal evolution in the ELISA-determined anti-PGL-I antibody isotype from predominantly IgM to IgG (Table 3). Many ML-inoculated RM failed to develop clinical symptoms of leprosy in the presence of significant IgG and absence of IgM anti-PGL-I antibody (nos 15-25, Table 3). The persistence of viable ML in the absence of clinical signs of infection in many of these RM was suggested by longitudinally stable high levels of antiPGL-I IgG antibody, as illustrated in Figure 1a-c by RM's D553, D739 and F183 (nos 2123, Tables $1 \& 3$ ). These longitudinal IgG anti-PGL-I profiles are very similar to RM with paucibacillary leprosy. Of the 38 animals studied immunologically, persisting IgG anti-PGLI antibody levels were observed in the absence of IgM anti-PGL-I antibody in 13 of the 24 RMs lacking clinical signs of leprosy (Table 3).

Of $24 \mathrm{RM}$ that failed to develop leprosy, 19 were examined both for anti-PGL-I antibody and for lepromin skin test responses (Table 3). Eleven (nos 26, 28-33, 35 \& 36-38) of the 19 $(57.9 \%)$ failed to produce greater than cut-off levels of either IgM or IgG serum antibody to PGL-I (Table 3). Of these 11 antibody-negative, leprosy-negative RM, eight were lepromin skin tested and six of the eight $(75 \%)$ were skin test positive (the remaining two were $+/-$ ). Seven of the eight antibody-negative, leprosy-negative, skin test-positive RM were also observed for in vitro blastogenic responsiveness to lepromin and only one (14.3\%) (F719, no. 36, Table 3) was positive. Among the 13 leprosy-negative IgG anti-PGL-I positive group, 
Table 3. Relationship in RM between clinical leprosy and anti-PGL-1 antibody, lepromin skin test results and in vitro blastogenic responses to lepromin

\begin{tabular}{|c|c|c|c|c|c|c|c|}
\hline \multirow[b]{2}{*}{ No. } & \multirow{2}{*}{$\begin{array}{l}\text { Animal } \\
\text { number }\end{array}$} & \multicolumn{2}{|c|}{$\begin{array}{c}\text { Anti-PGL-1 } \\
\text { Ab Isotype }\end{array}$} & \multirow[b]{2}{*}{ Clinical status } & \multicolumn{2}{|c|}{ Lepromin reactivity } & \multirow{2}{*}{$\begin{array}{l}\text { SIV } \\
\text { status }\end{array}$} \\
\hline & & IgM & $\operatorname{IgG}$ & & $\mathrm{ST}^{\mathrm{a}}$ & Blastogen ${ }^{b}$ & \\
\hline 1 & F185 & + & + & $\mathrm{LL}^{\mathrm{c}}$ & - & - & - \\
\hline 2 & A125 & + & - & $\mathrm{LL}$ & - & $\mathrm{ND}^{\mathrm{d}}$ & - \\
\hline 3 & F179 & + & + & Ind/TT, $L^{g}$ & $+^{\mathrm{e}}$ & - & - \\
\hline 4 & A491 & - & + & $\mathrm{BB}$ & +++ & ND & + \\
\hline 5 & E423 & - & + & $\mathrm{BB}$ & ++ & + & + \\
\hline 6 & 8664 & - & + & $\mathrm{BL}$ & - & ND & + \\
\hline 7 & F187 & + & + & LL, Ind/TT, neuritic ${ }^{\mathrm{h}}$ & + & - & - \\
\hline 8 & B539 & - & + & TT, neuritic & ND & ND & - \\
\hline 9 & B465 & + & + & Ind/TT & + & + & - \\
\hline 10 & A749 & - & + & Ind/TT & + & - & - \\
\hline 11 & F383 & - & + & Ind/TT & + & - & - \\
\hline 12 & C406 & - & + & Ind/TT & - & - & + \\
\hline 13 & B988 & - & - & Ind/TT & - & ND & + \\
\hline 14 & F714 & - & - & Ind/TT & +++ & - & - \\
\hline 15 & B630 & - & + & $N_{V L}{ }^{f}$ & + & ++ & - \\
\hline 16 & B685 & - & + & NVL & +++ & ++ & - \\
\hline 17 & B614 & - & + & NVL & +++ & + & - \\
\hline 18 & B960 & - & + & NVL & +++ & +++ & - \\
\hline 19 & B748 & - & + & NVL & + & ND & + \\
\hline 20 & B845 & - & + & NVL & + & ND & + \\
\hline 21 & D553 & - & + & NVL & +++ & - & - \\
\hline 22 & D739 & - & + & NVL & +++ & - & + \\
\hline 23 & F183 & - & + & NVL & ND & ND & - \\
\hline 24 & D449 & - & + & NVL & + & - & + \\
\hline 25 & E118 & - & + & NVL & + & - & + \\
\hline 26 & B245 & - & - & NVL & ND & ND & - \\
\hline 27 & B347 & - & - & NVL & ND & ND & - \\
\hline 28 & A766 & - & - & NVL & ND & ND & - \\
\hline 29 & B784 & - & - & NVL & ND & ND & - \\
\hline 30 & B849 & - & - & NVL & + & - & - \\
\hline 31 & D436 & - & - & NVL & + & ND & - \\
\hline 32 & D505 & - & - & NVL & $+1-$ & - & + \\
\hline 33 & F704 & - & - & NVL & + & $+1-$ & - \\
\hline 34 & F915 & - & - & NVL & +++ & - & - \\
\hline 35 & F596 & - & - & NVL & $+1-$ & - & - \\
\hline 36 & F719 & - & - & NVL & +++ & + & - \\
\hline 37 & F688 & - & - & NVL & + & - & - \\
\hline 38 & F762 & - & - & NVL & + & - & - \\
\hline
\end{tabular}

${ }^{a}$ ST, skin test (results according to WHO criteria).

${ }^{\mathrm{b}}$ Blastogen, blastogenesis SI.

${ }^{\mathrm{c}} \mathrm{LL}$, lepromatous leprosy; BB, borderline leprosy; Ind/TT, indeterminate/tuberculoid leprosy and TT, tuberculoid leprosy.

${ }^{\mathrm{d}} \mathrm{ND}$, not determined.

$\mathrm{e}_{+}++,+,+/-$; Lepromin skin test results 27 months after the first inoculation $(+++) ; 3$ months after a second inoculation (+) and 29 months after the second (SIV contaminated) inoculation (+/-).

${ }^{\mathrm{f}} \mathrm{NVL}$, no visible leprosy.

${ }^{\mathrm{g}}$ The initial disease in F179 was in the Ind/TT area of the spectrum (skin test and blastogenesis determinations were done during this period), but by approximately 8 years PI the disease evolved to the LL type.

${ }^{\mathrm{h}}$ The disease in F187 was initially LL (skin testing and blastogenesis were done at this time) but evolved to Ind/TT type involving dermal nerves. 
a. $\mathbf{0 7 3 9}$

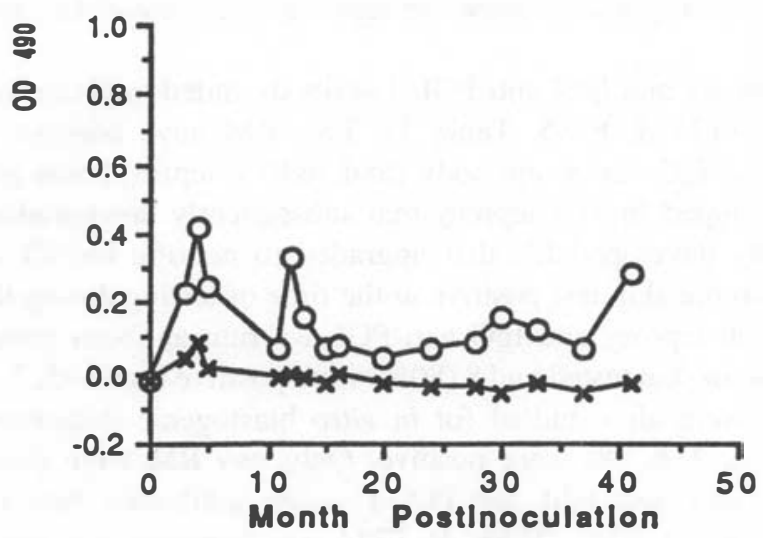

b. $\mathrm{D} 553$

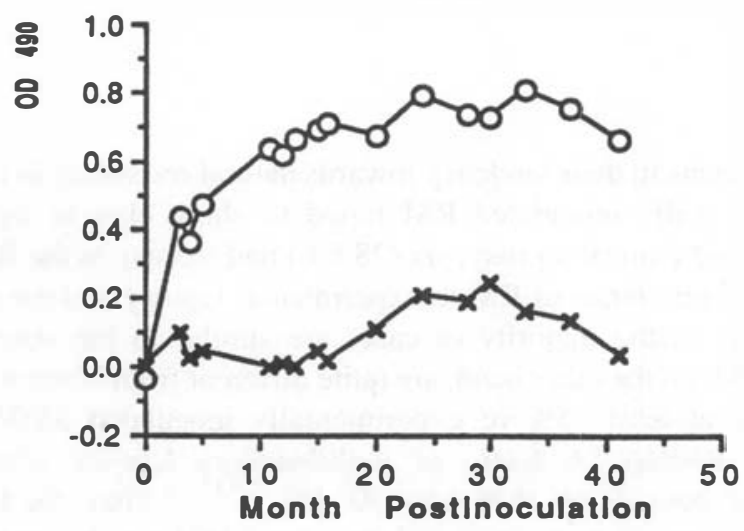

C. $\mathbf{F 1 8 3}$

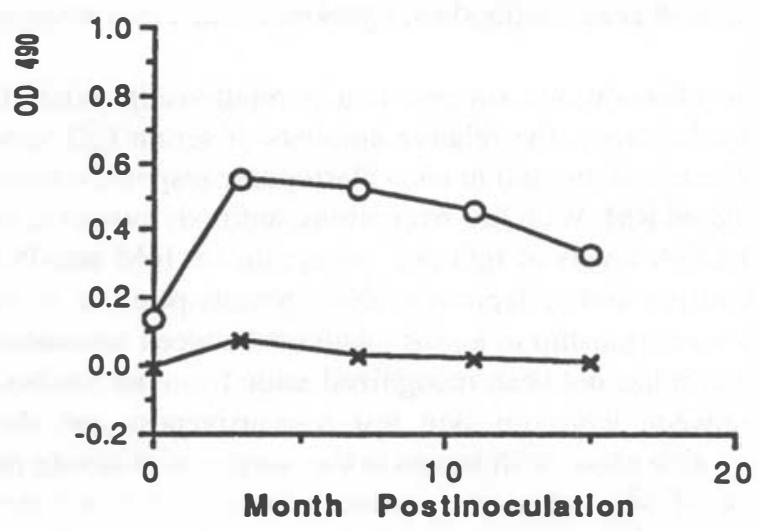

Figure 1. ELISA-determined anti-PGL-I $\operatorname{IgM}(\times)$ and $\operatorname{IgG}(\bigcirc)$ serum antibody profiles preinoculation and longitudinally after M. leprae inoculation in RM D739 (a), D553 (b) and F183 (c). 
11 were lepromin-skin tested and $100 \%$ were positive. Of these $11 \mathrm{RM}$, nine were also studied for in vitro blastogenic responses to lepromin and four (44\%) responded positively (Table 3).

RM with LL leprosy and IgM anti-PGL-I antibody failed to give positive lepromin skin tests (nos $1 \& 2$, A125 \& F185, Table 3). Two RM gave positive lepromin skin test responses at the time IgG serum antibody (and Ind/TT leprosy) was present: no. 3, F179 which initially developed Ind/TT leprosy that subsequently downgraded to LL and no. 7, F187 which initially developed LL that upgraded to neuritic Ind/TT (Table 3). Both of these RM were lepromin skin test positive at the time of testing during their Ind/TT disease period. Ten RM with leprosy and IgG anti-PGL-I serum antibody (nos 3-7, 9-12 \& 34, Table 3) were lepromin skin tested and $8(80 \%)$ were positive (nos 3-5, 7, 9-11 \& 34). Seven of these eight RM were also studied for in vitro blastogenic responsiveness to lepromin and two $(28.6 \%$, nos $3 \& 38)$ were positive. Only two RM were found to have clinical leprosy with both IgG and IgM anti-PLG-I serum antibodies below the cut-off point (no. 13, B988 and no. 14, F714, Table 3). F714 was lepromin skin test-positive and B988 was lepromin-negative.

\section{Discussion}

RM are similar to humans in their tendency towards natural resistance to leprosy in that 25 of $46(54.3 \%)$ experimentally inoculated RM failed to show clinical signs of leprosy and of the 21 that developed clinical leprosy, six (28.6\%) had leprosy in the BB-LL region of the spectrum. The natural resistance of RM to experimental leprosy and the propensity towards paucibacillary leprosy in the majority of cases are similar to the observations in human leprosy. Captive SMM, on the other hand, are quite different from humans and RM in leprosy susceptibility in that at least $75 \%$ of experimentally inoculated SMM (and two known natural cases ${ }^{3,13,17}$ ) develop LL forms of multibacillary leprosy when inoculated with the minimal infective dose of less than $4.8 \times 10^{7}$ ML. $^{3,9,11,12}$ Thus, the RM appears to be a more appropriate non-human primate model than the SMM for the study of leprosy. Both SMM and RM are similar to humans phylogenetically and human immunological reagents cross-react with lymphoid cells, antibodies, cytokines and other immune elements in both species. $3,6,9-13,18-23$

The immunologic observations suggest that a relationship exists between resistance/ susceptibility to clinical leprosy, the relative amounts of serum IgG versus IgM anti-PGL-I antibody, lepromin skin test status and in vitro blastogenic responsiveness to lepromin among experimentally inoculated RM. With few exceptions, antibody-positive, leprosy-resistant RM which had positive ELISA levels of IgG and no significant IgM anti-PGL-I antibody, were lepromin skin test-positive and/or lepromin blastogenesis-positive in vitro. It is generally accepted that there is a relationship in leprosy patients between resistance and lepromin skin test responsiveness, but it has not been recognized aside from our studies, to our knowledge, that a correlation between lepromin skin test responsiveness and the humoral immune response compartment also exists with regard to the isotype of antibody produced in response to the PGL-I antigen of ML. This relationship between CMI and the humoral immune compartment may be direct or coincidental, but appears likely to depend at least partially upon common or overlapping lymphokine pathways between the humoral and the CMI compartment. Thus, successful CMI in the RM, as manifested by skin test responsiveness 
and/or blastogenesis against ML antigens, correlates with the production of IgG but not IgM isotype serum antibody against the PGL-I ML cell wall antigen.

It is noteworthy that we observed that positive skin test responses do not always directly correlate with positive in vitro blastogenic responses to ML antigens, so that lack of an effective clinical response could be due to a failure of effector cells to migrate into the local dermal sites of ML growth or to a failure of effector cells to respond appropriately to ML antigens or both.

These and our previous observations in RM, SMM and chimpanzees suggest that CMI and anti-PGL-I IgG/IgM serum antibody production may be under similar or overlapping feedback controls, with IgG isotype being associated with increased and IgM isotype being associated with decreased anti-ML CMI. ${ }^{6,9,10,12,16}$ We do not imply that the antibody necessarily plays a direct role in the CMI responses or even in the elimination of the pathogen. We only note that an association between the anti-PGL-I antibody compartment and the anti-ML CMI compartment exists. In fact, 58\% of the leprosy-negative RM (11 of 19 studied) failed to produce significant levels of either IgG or IgM anti-PGL-I antibody responses; $67 \%$ of these antibody-negative, leprosy-negative RM that were also lepromin skin tested were lepromin-positive. Thus, it is possible to mount an effective CMI response to ML antigens capable of eliminating the pathogen in the absence of a significant detectable antibody response. It would be difficult or impossible to document such a relationship in humans, however, because of the long unrecognized incubation period and the fact that most of the changes noted in the immune status of non-human primate models take place early after infection and evolve to a less dynamic stage by the time clinical disease is diagnosed.

We demonstrated that some RM maintain an elevated or fluctuating level of IgG antiPGL-I antibody that can persist for years in the absence of visible clinical disease. These RM are believed to harbour viable 'persister' ML. Thus, it would appear that the persistence of anti-PGL-I antibody is a good indicator of the persistence of viable ML in the host; so long as that antibody is of the IgG isotype, little or no clinical progress of the disease appears likely. Such animals usually give a strong positive lepromin skin test and/or in vitro blastogenic responses.

Finally, the main strength of the RM model may be in the study of the immunological changes in conjunction with clinical implications in the understanding of the pathogenesis of leprosy early postinfection, a time period which is nearly impossible to study in humans. We do not know the significance of the association between the serum anti-PGL-I responses and the relative degree of susceptibility to the spectral forms of leprosy. It appears important to study this phenomenon in greater detail, however, since it may lead to the further definition of the role of lymphokines and other cytokines in overlapping immune pathways. Such information could lead to the discovery of as yet unrecognized and undefined areas of potential immunoprophylactic and/or immunotherapeutic intervention in leprosy and other mycobacterial diseases.

\section{Acknowledgements}

We are indebted to the following persons for technical assistance: Ms Cynthia Trygg, Ms Janelle LeBlanc, Ms Carol Coyne, Ms Doris O'Leary, Ms Eva Pecunia, Mr Calvin Lanclos and Ms Renee Grow. We thank Ms Ann Bennett for secretarial 
Assistance. This study was supported by NIH grants \#Al-19302 from the National Institute of Allergy and Infectious Diseases and \#RR-00164 from the National Center for Research Resources.

\section{References}

${ }^{1}$ Wolf RH, Gormus BJ, Martin LN, Walsh GP, Meyers WM, Binford CH. Experimental leprosy in three species of monkeys. Science, 1985; 227: 529-531.

2 Baskin GB, Gormus BJ, Martin LN, Wolf RH, Murphey-Corb M, Walsh GP, Binford CH, Meyers WM, Malaty R. Experimental leprosy in a Rhesus monkey: necropsy findings. Int J Lepr, 1987; 55: 109-115.

${ }^{3}$ Meyers WM, Gormus BJ, Walsh GP. Leprosy in nonhuman primates. In: Leprosy R C Hastings, ed. Churchill Livingston, London, 1994: 385-408.

4 Martin LN, Gormus BJ, Wolf RH, Walsh GP, Meyers WM, Binford CH. Experimental leprosy in nonhuman primates. Adv Vet Sci Comp Med, 1984; 28: 201-236.

5 Baskin GB, Gormus BJ, Martin LN, Murphey-Corb M, Walsh GP, Meyers WM. Pathology of dual Mycobacterium leprae and simian immunodeficiency virus infection in Rhesus monkeys. Int J Lepr, 1990; 58: $358-365$.

6 Gormus BJ, Murphey-Corb M, Martin LN, Zhang J, Baskin GB, Trygg C, Walsh GP, Meyers WM. Interactions between simian immunodeficiency virus and Mycobacterium leprae in experimentally inoculated Rhesus monkeys. J Infect Dis, 1989; 160: 405-413.

7 Shepard CC, McRae DH. A method for counting acid fast bacteria. Int J Lepr, 1968; 36: 78-82.

8 Ridley DS, Jopling WH. Classification of leprosy according to immunity: a five group system. Int J Lepr, 1966; 34: $255-273$.

${ }^{9}$ Gormus BJ, Ohashi DK, Ohkawa S, Walsh G, Meyers WM, Brennan PJ, Trygg C. Serologic responses to Mycobacterium leprae-specific phenolic glycolipid I. Antigen in sooty mangabey monkeys with experimental leprosy. Int J Lepr, 1988; 56: 537-545.

10 Baskin GB, Gormus BJ, Xu K, Martin LN, Wolf RH, Cantrell C, Pezeshkpour GH, Walsh GP, Malaty R, Meyers WM. Lepromatous leprosy with intraneural Erythema nodosum leprosum in a mangabey monkey. Int J Lepr, 1991; 59: 618-623.

${ }^{11}$ Gormus BJ, Xu K, Meyers WM, Walsh GP, Levis WR, Meeker HC. Antibodies to lipoarabinomannan antigen in sooty mangabey monkeys experimentally inoculated with Mycobacterium leprae. Int J Lepr, 1990; 58: $65-72$.

12 Gormus BJ, Xu K, Baskin GB, Bohm RP, Jr, Blanchard JL, Mack PA, Ratterree MS, McClure HM, Meyers WM, Walsh GP. Experimental leprosy in monkeys. II. Longitudinal serological observations in sooty mangabey monkeys. Lepr Rev, 1995; 66: 105-125.

13 Meyers WM, Walsh GP, Brown HL, Binford CH, Imes GB, Hadfield TL, Schlagel CJ, Fukunishi Y, Gerone PJ, Wolf RH, Gormus BJ, Martin LN, Harboe M, Imaeda T. Leprosy in a mangabey monkey: naturally-acquired infection. Int J Lepr, 1985; 53: 1-14.

14 Fultz PN, Gordon TP, Anderson DC, McClure HM. Prevalence of natural infection with simian immunodeficiency virus and simian T-cell leukemia virus type I in a breeding colony of sooty mangabey monkeys. AIDS, 1990; 4: 619-625.

15 Murphey-Corb M, Martin LN, Rangan SRS, Baskin GB, Gormus BJ, Wolf RH, Andes WA, West M, Montelero RC. Isolation of an HTLV-III related retrovirus from macaques with simian AIDS and its possible origin in asymptomatic monkeys. Nature, 1986; 321: 435-437.

16 Gormus BJ, Xu K, Alford P, Lee DR, Hubbard G, Eichberg JW, Meyers WM. A serologic study of naturallyacquired leprosy in chimpanzees. Int J Lepr, 1991; 59: 450-457.

17 Gormus BJ, Wolf RH, Baskin GB, Ohkawa S, Gerone PJ, Walsh GP, Meyers WM, Binford CH, Greer WE. A second sooty mangabey monkey with naturally acquired leprosy: first reported possible monkey-to-monkey transmission. Int J Lepr, 1988; 56: 61-65.

18 Gormus BJ, Xu K, Baskin GB, Bohm RP, Jr., Blanchard JL, Mack PA, Ratterree MS, McClure HM, Meyers WM, Walsh GP. Experimental leprosy in monkeys. I. Sooty mangabey monkeys: transmission, susceptibility, clinical and pathological findings. Lepr Rev, 1995; 66: 96-104.

19 Martin LN, Gormus BJ, Bozelka BE. Functional analysis of monkey lymphocyte subsets defined by OKT4 and OKT8 monoclonal antibodies. Cell Immunol, 1983; 77: 338-347.

20 Martin LN, Gormus BJ, Wolf RH, Gerone PJ, Meyers WM, Walsh GP, Binford CH, Hadfield TL, Schlagel CJ. Depression of lymphocyte responses to mitogens in mangabey monkeys with disseminated experimental leprosy. Cell Immunol, 1985; 90: 115-130.

21 Ohkawa S, Fukunishi Y, Martin LN, Gormus BJ. Regulatory role of FcR+ and FcR- Monocyte subsets in Mycobacterium leprae-induced lymphoproliferative responses of in vitro. Clin Exp Immunol, 1987; 67: 43-50. 
22 Ohkawa S, Martin LN, Gormus BJ. Lymphoproliferative responses of experimental leprosy monkeys: regulatory role of monocyte and lymphocyte subsets. J Immunol, 1987; 138: 3943-3948.

${ }^{23}$ Gormus BJ, Martin LN, Baskin GB, Wolf RH, Meyers WM, Walsh GP, Binford CH. Experimental leprosy in nonhuman primates - current status. In: A window on leprosy, BR Chatterjee, ed. Statesman Commercial Press, Calcutta, 1993: 554-568. 